\title{
Einfluss von Beihilferichtlinien auf die private Zahnheilkunde?
}

\author{
Eine Bestimmung lautet: „Der Ansatz der Nummern 15, 30, 31 und 34 GOÄ ist im \\ Zusammenhang mit zahnärztlichen Maßnahmen grundsätzlich nicht gerechtfertigt." Die Ziffern \\ Ä15 (Einleitung flankierender Therapie bei ambulanter Chronikerbetreuung), Ä30, Ä31 (Homöopathie- \\ anamnese und -behandlung) und Ä34 (Erörterung der Auswirkungen bei nachhaltig lebensverän- \\ dernder Erkrankung) sind gemäß novelliertem § 6 (2) GOZ ausdrücklich für Zahnärzte ansetzbar.
}

Aber warum wohl hat der Verordnungsgeber diese Leistungen ausdrücklich für Zahnärzte zugänglich gemacht? Weil er den Ansatz dieser Leistungen im Zusammenhang mit zahnärztlichen Maßnahmen grundsätzlich als nicht gerechtfertigt sieht? Wohl kaum. Die „Beihilfebeurteilung“ dieser Gebührenziffern geht entschieden zu weit:

Spezielle Beratung in GOZ/GOÄ ist niemals abgegolten mit allgemeiner Beratung. Spezielle Beratung (Führung, Anleitung, etc.) folgt erst auf allgemeine Beratung.

Als Beispiel soll die Ä34 näher betrachtet werden. Deren umfangreicher Wortlaut ist zum Teil alternativ angelegt im Sinne von „entweder oder“, das heißt Ausschluss des einen oder anderen Bestandteils der Leistungsbeschreibung im konkreten Fall. In der Zahnmedizin könnte also eine verkürzte Bezeichnung so formuliert werden: „Erörterung (mindestens 20 Minuten) der Auswirkung einer nachhaltig lebensverändernden Erkrankung auf die Lebensgestaltung bei Feststellung der Erkrankung“.

Der $\$ 10$ (2) GOZ sieht statt Angabe der vollständigen „Leistungsbeschreibung“ auf der Rechnung die Angabe einer zutreffenden „Bezeichnung“ vor. Die plumpe Ablehnung, die Erkrankung sei nicht „lebensbedrohlich“, wird in letzter Zeit nur noch selten vorgebracht.

Was wären denn nachhaltig lebensverändernde Erkrankungen in der Zahnmedizin oder mit Auswirkungen auf den zahnmedizinischen Bereich? Da kämen zum Beispiel agressive (juvenile) Parodontitis (profunda) und dekompensierte CMD (kraniomandibuläre Dysfunktion) in Frage, gegebenenfalls eine Operation erfordernde Progenie oder Mikrogenie, Aplasien, aber auch Allgemeinerkrankungen wie etwa Osteoporose, Diabetes oder Morbus Crohn.

Ein Rat zur Berechnung der kaum delegierbaren Ä34 mit mindestens 20 Minuten Dauer: Mit dem Faktor 3,5 werden betriebswirtschaftlich allenfalls 15 Minuten zahnärztliche Zeit abgegolten. In der dann erforderlichen Begründung für die Gebührenhöhe kann man den medizinischen Grund für den An- satz der Leistung zusätzlich nennen und erspart sich wohlmöglich honorarverkürzenden Schriftverkehr.

\section{Beihilfebestimmung nur für die Beihilfe selber?}

„Im Hinblick auf die Rechtsprechung des Bundesgerichtshofs (Urteil vom 13.10.2011 - III ZR 231/10) ist spätestens vor einem Klageverfahren eine amtszahnärztliche Meinung einzuholen und zu den Akten zu nehmen." Diese erstaunliche Beihilfebestimmung ist eine Zumutung für Beihilfeberechtigte. Wenn man das angesprochene Urteil des Bundesgerichtshofs kennt und seinen Inhalt, nämlich Verurteilung einer Beihilfestelle zu Schadenersatz wegen Amtspflicht verletzender, pauschaler Ablehnung von Begründungen, dann erstaunt die Reaktion in der Beihilfebestimmung umso mehr. Die sagt letztendlich: weitermachen wie bisher und erst dann, wenn wirklich eine Klage droht, die gerichtlich geforderte Hinzuziehung von Sachverstand veranlassen. Beileibe aber nicht die vom BGH in erster Linie angesprochene Zahnärztekammer, auch nicht den an zweiter Stelle genannten „Sachverständigen“, sondern gemäß Beihilfebestimmung nur den Amtszahnarzt - natürlich lediglich mit seiner „Meinung“, nicht mit einem Gutachten, denn das wäre ja gemäß BGH (11.06.2003, Az. IV ZR 418/02) vorlage- und aushändigungspflichtig.

(c) ZA eG, P.E.

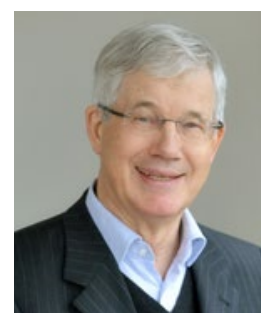

Dr. Peter H.G. Esser

GOZ-Experte und -Berater der ZA eG www.za-abrechnung.de 